OOMPUTATIONAL MATHEMATICS

BANACH CENTRR PUBLIOATIONS, VOLUME 13

PWN-POLISH SCIENTIFIO PUBLISHERS

WARSAW 1984

\title{
ПАКЕТ ПРИКЛАДНЫХ ПРОГРАММ САФРА
}

\author{
M. М. ГОР ВУНОВ.ПОСАДОВ, В. Я. КАРПОВ, Д. А. КОРЯГИН \\ Пиститут Прикладной Математики ия. М. В. Нелдыща АН ССCP, \\ Mocrea, CCCP
}

\section{1. Программное обеспечсиие вычислителыного эксперимента}

В настоящее время одним из основных методов изучения сложных фиаических и инженерно-технических проблем является метод вычислительного әксперимента, т.е. моделирование интересуюшего исследователя процесса на вычислительной машине.

Для физидеских задач основные этапы вычислительного эксперимента состоят в следугшем:

1. Выбор физического приближения и формулировка математической модели (как задачи математической физики).

2. Выбор дискретной модели, аппроксимирующей исходную математическуго задачу (например, построение разностной схемы) и разработка вычислительного алгоритма для решения уравнений.

3. Создание программы для реализации вычислителыного алгоритма.

4. Проведение расчетов и обработка полученной информации.

5. Анализ результатов, сравнение с физическим әкспериментом, пересмотр и уточнение физической модели п, если нунно, повторение всех этапов сначала.

С точки зрения программирования вычислительный эксперимент характерен тем, что для кандой физическоп̆ модели с целыо установления соответствия между физическим әкспериментом и вычислительным әкспериментом необходимо решать большое число вариантов (варьируя определяющие параметры задачи) и, кроме того, менять (Уточнять) саму физическуг модель. Эта особенность („многовариантность" и „многомодельность") вычислительного эксперимента проявляется в многократных изменениях программы, реализующей вычислительный алгоритм, причем эти изменения гасалотся кал структуры 
программы в целом, таг и отделыных фрагментов программной реализации алгоритма.

Фактически, разработка программы для вычислитсльного эксперимента выливается в создание крупной программной системы (обтемом порядка $10^{5} \div 10^{6}$ команд), хараттеризугщейся большим числом гомпонент и многообразием их взаимодействия. По-видимому, едигственшый реалынй путь обеспечения вычислительного эгсперимента программными средствами, позволягщими быстро собирать программы и моделировать вычислительный алгоритм и физическую модель, загллотается в использовании модулыного гринципа программирования в сочетании с раввитыми системными средствами. Плодотворность модульного принципа программирования обеспечивается тем ванным обстоятельством, что описание одпих и тех же физических процессов входит в различные комплексные проблемы. Кроме того, различные физитеские процессы часто описыватотся одними и теми भее уравнениями. Например, одними и теми же уравнениями описызатотся тание процессы гак дифф̆узия, теплопроводность, намагничивание. Различис между процессами проявляется лишь в физичесгом смысле гоэффициентов уравнений и искомой функции.

Что касается системных средств, то можно сказать, что разрабатывавшиеся до спх пор системные средства, несмотря на их прагтическую направленность, кат правило, были ориентированы на пеғоего "обобщенного" пользователя. Әто, конечно, обеспечивало их весьма широгуго применимость, но в то же время приводило I тому, что с тохпи зрения каждой жонгретной прикладной деятелыности они оказывалхсь не вполне адекватными. Иначе говоря, возмонносги, реализуемые системными средствами, определялись исходя из учета общих, а не специфических прикладных интересов. По мере расширения сфер прикладного программирования й соверценствования его методов выяснилось, что услуг, обеспечиваемых универсальными системными средствами (например, средствами отладкіи) не достаточно для успешного проведения работ, а такяе, что более характерно, испольвование татих средств для специальных целей (в qастности, редагтировагин, модификации х сборки программ) сопряжено с трудоемкими операциями. На наш взгляд, это следствие сепаратизма, присущего разработкам прикладного и системного программироватия, па современном әтапе становится тормозом развития махшиных припожений, связанных с решением гомплексных проблсм.

Проведенные рассмотрения позволяпт сделать вывод о том, что одним нз главных гаправлений работ в современном программировании должно стать создание проблемно-ориентированных программных систем, называемых пакетами пригладных программ. Причем успех на этом направлении мояет быть достигнут только путем объе- 
динения усилий толлективов прикладных и системных программистов.

\section{2. Функциональное и системное наполнения пакета прикладных программ}

Под пакетом прияладных программ мы понимаем комплекс вәаимосвязанных прикладных и системных программ, обсспечивающих адекватное покрытие шекоторо прйлгадной деятельности. Поясним несколько подробнее использованные здесь термины.

Прежде всего отметим, что всякая конкретная прикладная деятельность харагтеризуется цвумя фагторами. Во-первых, предметной областьг, то есть тем, на что направлен труд ирограммиста, представляющую собой совокупность решаемых им прикладных задач, In, во-вторых, дисциплиной работы, т.е. совонупностьо приемов, правил, принятых при разработке, отладке и эксплуатации программ. Адекватность покрытия означает достаточную полноту средств, связанных как с первым, так и со вторым фагторами, определяющими конкретную прикладную деятельность. Другими словами, достаточно полным должен быть как состав модулей, служащих материалом для сборки программ из данной предметной области, так и состав средств хранения, редактирования модулей, сборни программ и выполнения других видов принятых в данной прикладной деятельности работ. При этом в общем случае адекватность покрытия следует рассматривать во времсни, то есть всякое изменение в прикладной деятельності должно учитываться путем вилючения в пакет новых прикладных или системных программ. Таким образом, можно говорить о „динамической" адекватности покрытия.

Рассмотрим в качестве примера Систему Автоматизации Физических Расчетов (согращенно САФРА), созданную в Институте прикладной математики им. М. В. Келдыша АН СССР. Этот пакет в существуощем сейчас состоянии предназначен для расчетов задач одномерной газовой динамини с теплопроводностыо с учетом различных физических процессов, тагих нак магнитные поля (задачи о пинчах), двухтемпературность, перенос нэлучения, поглощение света лазера, учет термоядерных реакций (әадачи лазерного термоядерного синтеза). Всё это огредсляет предмстпую область пакета САФРА.

Кроме предметной области в пакете зафиксирована определенная технология разработки и эксплуатации программ решения больших физических задач. Эта технология выбрапа на основе изучения предшествующего опыта создания тагих программ и, в частности, опыта разработки и эксплуатации программ с помоцыо системы OLYMPUS, 
разработанной под руководством догтора Робертса в Калэмсной лаборатории (Велитообритания).

Характерной особенностыо работ, связанных с программами решения этих задач, является отсутствие чётко выраженно и хронологичеслой границы между әтапами разработки и эксплуатации татих программ. Кроме того, содержанию работ на обоих этапах присуща известная технологическая общность. В связи с этим средства пакета САФРА выбраны таким образом, что они в равной мере могут быть использованы как при разработке, так и при эксплуатации программ.

Таним образом, организационно пагет прикладных программ монкіо представить состоящим из двух пастей: функционального и системного наполнений.

Функциональне наполнение отражкет специфику предметпой области пакета и внлючает, например:

- совокупность модулей, используемых при составлении программ для решения задач данной предметной области;

- набор стандартных схем счета, определягщих модули, из готорых состоит программа решения той или иной типичной задачи;

- набор описаний, отражагщих различные функциональные ит программно-эксплуатационные характеристики модулей и стандартных схем счета, входящих в пагет.

Требования на форму представления и органиғацио элементов фунциионального наполнения устанавливаготся обычно при определении системных средств пакета.

Системное наполнение является административным органом пакета, отражающим дисциплину работы с пакетом. Системное наполнение может вилючать, например, такие компоненты:

- язык заданин, являгщийся средством общения пользователя с пагетом;

- архив, являгщийся системоц хранения элементов фуннционального наполнения и служебной информации пакета;

- монитор, представляющий собой совокупность программных средств, обеспечивапщих операционные возможности пакета.

Следует отметить, что системное наполнение может быть в известной степени инвариантным относительно гредметной области, то есть системное наполнение, разработапное для некоторой предметной области, может оказаться легко адаптируемым для других предметных областей.

Рассмотрим подробнее основные гомпоненты пакета САФРА.

\section{3. Архив пакета САФРА}

Архив предназначается в первуго очередь для хранения накапливаемого программного фонда и формулируемой на яәыке заданий ин- 
формации о структуре собираемых программ. Хранимые в архиве единицы информации называются коду.лями пакета и являготся основными элементами обработки в пакете прикладных программ САФРА. Кажњдый модуль харагтеризуется наличием у него архияного имени и вовможностью независимого доступа. Всяний доступ из задания пользователя $\kappa$ модулю в архиве осуществляется толыко посредством указания в задании архивного имени этого модуля, т.е. имени, под которым әтот модуль хранится в архиве. Специфика проблем сборки и стремление $\mathrm{k}$ логической простоте и экономичности языка заданий предопределили отход от традиционного понимания модуля, которое обычно ассоциируется с законченной программной единицей, допускатоце fí автономнуго трансляцию. В системе САФРА в модули выделяются функционально связные части программируемого алгоритма илі задания на сборку вне зависимости от того, будут. ли они допускать автономную трансляцио или пет. Архивное имя модуля явплется важным объектом в системе САФРА, поскольну после опредсленных автоматических преобразований задание на сборку сводится If перечислепио в нужном порядге архивных имен модулей, из которых составляются программа и ее начальные данные. Гибкость использования программного фонда и удобство широгого варьирования сочетани модулей в программах основывапося на том, что в программах па языке заданий указываптся не архивиые имена модулей, с готорыми требуется установить программные связи, а фунтциональные имена, отраянаюцие функциональный смысл того илю пного пужного модуля и определяющие место такого модуля в общей структуре программы. Гандое функциональное имя относится к нелоторому глассу модулей, имеющих сходное значепие. В языне эаданий предусмотрены средства указания соответствий между функциональными и архивными именамп модулей для задания тонкретных вариантов сборни.

В системе САФРА различаготся модули следующих типов:

1. Программная единица (PRUNIT),

2. Вставка (MAORO),

3. Схема счета (SCHEME),

4. Версія (VERSION),

5. Bapuart (VARIANT).

Модуль программная единица слунит апалогом программной единицы язына программирования. (Примерами программных единиц являются основная программа, подпрограмма либо фуниция-подпрограмма языка ФОРТРАН, процедура модульных версий языка АЛГОЛ и т.п.). В отличие от программной единицы языка программирования, модуль пакета может содержать не только предложения языка прог- 
раммирования, но и спепиальные управлялщие предпояепия языка заданий пакета, ғоторые, папример, могут угазывать на то, что в данное место модуля нунно вставить пекоторуг последоватсльность предлонкенй, готорая в пањете описана в виде модуля вставки.

Модуль вставка предназначается для оформления одного или нескольких предложений языка программирования или языка заданий, готорые могут слуяить вставками, ғапрнмер, в программные единицы пли в модули других типов. Обычно в виде вставок оформляотся последовательности операторов, имегцие относительно самостоятелыне значене и определяпцие те лоналыные решени, ноторые могут изменяться в различных программілх гомбннапиях. Например, для ФОРТРАІ-'а имеет смысл ог)рормляты в виде вставок изменяемые в зависимости от масштабпости решасмой задачи операторы DIMENSION или OOMMON. Посгольку на одну и ту же вставюу разрешается ссылаться из различных модулей пакета, то сопоставление одному фунцциональному имени вставки нового архивного нмени позволяет без дополнительных затрат труда пронзвести на уровне сборки нужнуг модификацию всех модулей, окончательный вид ноторых зависит от данного локального решения. В модуле вставка могут содержаться ссылии на другие вставки. Вставка не долякпа явно или неявно через цругие вставки ссылаться на себя.

Модуль схема счета предназначен для запоминания перечня основных фунюциональных элементов, на ноторые расчленяется алгоритм. Этому перечно элементов соогветствует набор программных единиц, участвугших в решении неготорого класса задач. Примером тагого набора с фигсированным порядком вызовов может слунить схема счета системы OLYMPUS. Схема счета составляется в терминах фугкциональных имен в расчете на широгую свободу варьирования нонкретных программ в рамках задаваемого перечня общих для этих программ функциональных элементов. При сборке гонкретной программы гаждому функционалыному имени ставится в соответствие некоторое архивное имя модуля. Аналогичная принципиальной блоксхеме в содержательных наименованиях, схема счета хранится в архиве для того, чтобы на ее основе системой могли пользоваться различные ліоди, работа которых сводится н внссенио в эту схему необходимых для конгретной реализации уточнений.

Модуль версия служит для описания гонкретной версии программы, т.с. для установления соответствия между отделыными функционалыными именами, указанными в схеме счета, и архнвиыми именами соответствугших программных единиц.

Модуль вариант предназначен для описания гонретного счетного варианта программы. В нем долнны быть нонгетизированы все 
всташки в программу, данные, необходимые для работы операторов ввода, ресурсы, требуемые программой, и т.д.

\section{4. Дисциплина работы в пакете САФРА}

Рассмотрим основныс моменты в подходе к разработке и эксплуатации программ при использовании пакета САФРА.

Предполагается, что разработка программы начинается с составления общей схемы счета, которая представляет собой перечисление функциональных имен модулей, отражающих основныс этапы решения задачи. Например, в системе OLYMPUS для решения задаq эволюционного типа зафиксирована схема счета, представленная на рис. 1.

\section{COTROL}

\section{DATA}

INITAL

OOTPUT(1)

STEPON

OUTPUT(2)

TESEND

ENDRUN

\section{STEPON}

STIT

MOTION

ENERGY

FIELD

CVERGE

Pис. 1. Схема счета

COTROL есть программная единица, осуществлягщая управление всем расчетом. Она последователын вызывает подпрограмму DATA задания основных параметров конкретного варианта;

- подпрограмму INITAL расчета физических величин на первом временном слое;

- полпрограмму OUTPUT со значением параметра-единица для выдачи на печать значений начальных данных.

Затем вызывается подпрограмма STEPON, результатом работы тоторой должно быть значение функций на новом временном слое. 
После того, кағ функции па новом временном слое вычислены, вызовом OUTPUT(2) производится пошаговая выдача данных и подпрограммой TESEND проверяется, нулкн ли продолжать расчет, или пужно его закончить. В первом случае производится возврат на STEPON, т.е. переход i расчету нового временного слоя, а во втором случае оформляется онончание расчета.

Каягдая из указанных выше программных единиц может обращаться if другим подпрограммам. Например, в задачах магнитно гидродинамики для растета решения на новом временном слое (подпрограмма STEPON) мы должны решить последовательно уравнение движения MOTION, уравнение энергии INERGY, уравпение поля FIELD и проверить CVERGE после этих расчетов, сошлись ли итерации. Если итерации не сошлись, мы снова последовательно вызываем указанные подпрограммы, а в прогивном случае подпрограмма STEPON свого работу заканчивает.

Таким образом, в процессе разработки внутренпей сгруктуры отдельного модуля в нем выделяются такие относительно самостоятельные qасти, которые предполагается оформить тоже в виде модулей. Таное выделение и накопление модулей различных уровней называется модуляризацией программы. При составлении программы ив модулей обращения к модулям могут оформляться двумя различными с технологпческой точни врения способами. Первый способ предполагает обрапепие if модулю c помолцьо соответствующей гонструкции языжа программирования, осуществлялощей вызов подпрограммы (например, оператором CALL), и в этом случае вызываемый модуль оформляется как самостоятельная программная единица (например, SUBROUTINE). При втором способе обращение оформляется в виде специалынй конструцции, являющейся по существу матровызовом. При таком способе вызываемые модули оформляготся гак вставни (магроопределения). Выбор конкретного способа оформления делается исходя только из сообранкений удобства и эффективности программной реализации. Работы по отладке программы могут вестись одновременно в пескольких направлениях, гаждое иэ которых, по существу, связано с отладкой одного или группы модулей пз числа определеных па данный момент в результате модуляризации программы. Каждому направлению ставигся в соответствие версия программы, хюторая представляет собой перечень соответствий между фунгционалыными именами модулей и архивными именами их реализаций. В число этих реализаций па стадии разработки программы могут входичь модули двух типов. Во-первых, „реальные" модули, полностыо отвечалощие фунгциональному назначению, ассоциируемому с данным фунгциональным именем, и, во-вторых, „имитирующие" модули, отвечагщие фупкциональному назначению лишь 
в той мере, в кағой это диктуется соображениями отладки. При этом этап разработки програмиы заключается в создании в архиве „реальпого" модуля, отвечатщего некоторому функциональному нмени, прнчем для одного функционального имени может быть полуяено несколько различных по программной реализации модуле̨й. Такая организация разработкц дает возможность, по-существу, исключить этап автономной отладки модулей, поскольну наличие в версии зафиксированных соответствий между функциональными и архивными именами обеспечивает имитацию работы отлаживаемого модуля в контексте всей программы. Логическим завершением этого процесса, напоминалшего программирование задач по принципу „сверху вниз", является получение версии или верспй, в готорых не будет ссылок на „пмитирующие" модули. Если такая версия не содержит свободных фунцциональных имен, то совокупность упоминаемых в ней архивных имен определяет единственный эксплуатационный вариант программы. Если же в верспи содержатся свободные функциональные имена, то с ее помощью задается некоторый класс эксплуатационных вариантов программы. Каждый из этих вариантов получается путем задания определенной совонупности архивных пмен, соответствующих свободным функциональным именам в версии.

\section{5. Язык заданий}

Работа с пакетом ведется посредством выполнения задания, написанного польљователем на языке заданий. Задание состоит из совокупности пунктов задания, выполняемых последовательно и определягоих такие действия, как

- внесение изменений в архив (запись п псклочение модулей);

- препроцессирование (т.е. предварительнуго обработку) текстов отделыпы модулей путем замены ссылок на вставки соогветствуощими текстами вставок;

- трансляцио модулей;

- сборку готовой программы из транслированных модулей;

- псредачу программы на счет;

- а также пекоторые другие виды работ.

Каждый пункт задания состоит из заголовна пункта задания либо нз заголовка пунгта и тела пупкта задания.

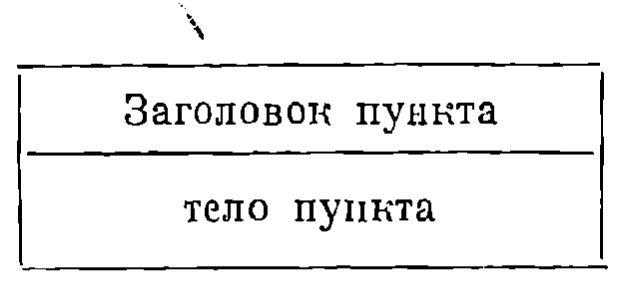


Заголовок пункта представляет собой строну (перфокарту), содержкашуо до 80 символов и имегощуо слгдугщий формат записи:

$$
\left\langle\begin{array}{c}
\text { признак } \\
\text { эаголовка }
\end{array}\right\rangle\langle\text { операция }\rangle\left\langle\begin{array}{c}
\text { тип } \\
\text { модуля }
\end{array}\right\rangle:\left\langle\begin{array}{c}
\text { архивное имя } \\
\text { модулл }
\end{array}\right\rangle .
$$

Признак заголовна начинается с первой позиции строк. Призиак заголовла должен быть одинатовым для всех пунктов одного задания н может меняться от задания ж заданию. Признаком заголовга монет слунить любая последовательность длиной не более трех символов, составленная из следугощих символов:

$$
+,-, *, 1
$$

Признак заголовна пс доляен содержать пробелов. Далее в настоящем описании в качестве признака заголовга мы используем символ + . Принципиальная возможность разнообразия признатов вводится для того, чтобы можно было отличать заголовки пунктов языка заданий от других предложенин в рамгах кодировки, принятой в той нли иной вычислителыной системе.

После признака заголовка может слецовать произвольное чпсло пробелов (в частности, ни одного), затем следует тип пунита, угазывающий операциг, для выполнения тоторой слуиит данный пункт задания, далее следует один или несколько пробелов или запятых, затем указываетсл тип модуля, над тоторым долкпа быть проведена данная операция, затем символ двоеточие и после него угазывастся имя модуля. Позиции строки с номерами 73-80 игнорируются. Архивным именем модуля может служить идентификатор, т.е. лобая комбинация из букв или цифр длиной не более шести символов, начинающаяся с буквы. В имени не могут содержаться пробельы.

Тело пункта - это последовательность предложений языка заданий, либо другая информация, заглюченная между заголовгом данного пунгта и заголовтом следуноцего пуннта, пли нонцом әадания, если данный пушет последний.

В любом месте задания могут быть записаны предлонсния = номментарии. Они имегот следующий формат записи

$$
\left\langle\begin{array}{c}
\text { признак } \\
\text { заголовка }
\end{array}\right\rangle \mathrm{C} \quad\langle\text { телст }\rangle \text {. }
$$

Комментарии могут служить для пояснения отдельных копструкций вадания и не оказывают никакого влияния на его выполненше. Ifoмментарии, встретившиеся в теле пункта операции STORE, предиазначенного для записи в архив нового модуля, запоминалотся іг воспро- 
изводятся при распечатке тенста данного модуля, но исключаготся при сборке из модулеіи тонкретной задачи.

Протоколом выполнения задания является его листинг, печаталощийся на АЦПУ. Если в процессе анализа некоторого пуніта выявляется кагая-либо ошибка, в листинге петатается соответствующее сообщение, и, в завнсимости от серьезности ошибки, отключается выполнение данного п части последующих пунктов. Для наждого из невыполненных пуннтов при әтом печатается сообщение:

\section{!!!!! БЫЛШ ОШИБКИ - ПУНКТ НЕ ВЫПОЛНЕН}

C другой стороны, после каждого выполиенного пунита печатается сообщение о результате его работы, папрпмер:

$$
+++++ \text { НОВЫЙ МОДУЈІ ДИСПАК СОЗДАН }
$$

ИJII

$$
+++++ \text { СФОРМИРОВАНА ЗАДАЧА ШИФР } 031100
$$

\section{Занести в архив новый модуль. (Операция STORE)}

Если пользователь хочет завести в архиве новый модуль определенного типа с определенным архивным именем, он должен указать об этом системе. Для этой цели слуюнт пуннт задания, заголовок которого пмеет вид

$$
+\operatorname{STORE}\left\langle\begin{array}{c}
\text { тип } \\
\text { модуля }
\end{array}\right\rangle:\left\langle\begin{array}{c}
\text { архивное имя } \\
\text { модуля }
\end{array}\right\rangle
$$

Телом данного пуикта задания является телст создаваемого модуля.

В результате выполнсния данного пунгта задапия в архиве появится модуль указанного в заголовке пункта типа с данным архнвным именем. Записанный в архив модуль можно пспользовать в последующих пунктах того же задания и в последующих заданиях. Любые использованпя этого модуля осуществляются путем ссылки на его архивное Iмн.

Tиi модуля в заголовке пунта STORE может быть опущен. В этом случае считается, что создаваемый модуль имеет тип MACRO.

Прпяер. Занесение в архив программной единицы.

+ STORE PRUNIT: OLMU 04

SUBROUTINE RVAR (KNAME, PVALUE)

$\mathrm{C}$

С 0.4 ПЕЧАТЬ ИДЕНТИФИКАТОРА IИ ЗНАЧЕНИЯ

O ПЕРЕМЕННОЙ 
C

COMMON /COMBAS/

1 ALTIME, CPTIME, NLEDGE, NLEND, NLRES, NONLIN,

2 NOUT, NPRINT, NREAD, NREO, NRESUM, NSTEP LOGICAL

1 NLEND, NLRES

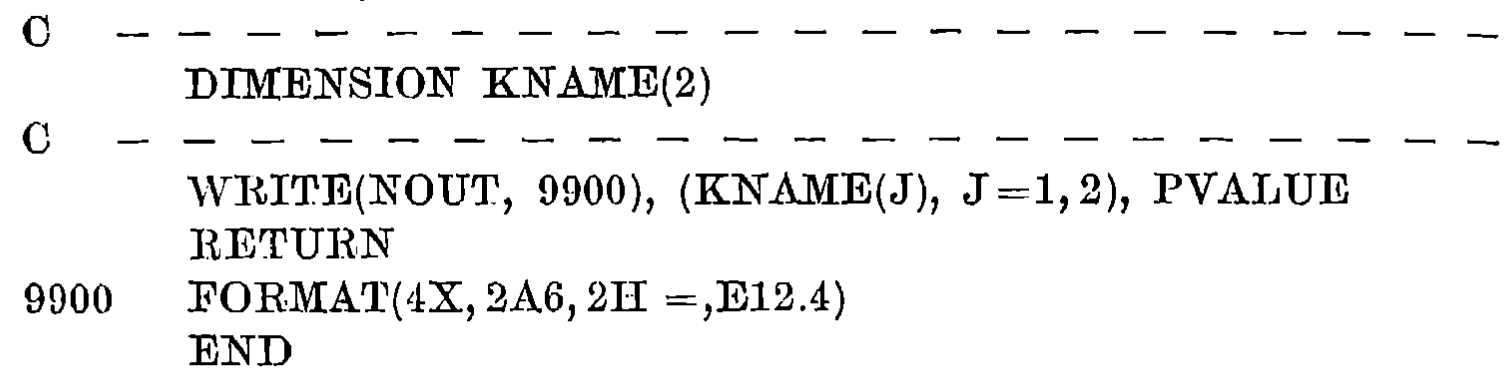

В реаультате выполнения этого пуншта задания в архив занесется модуль типа программная единица с именем OLMU 04, тело которого представляет собой фортранную подпрограмму с именем RVAR.

Исключить модуль из архива. (OMерация DELETE)

Операция DELETE позволяет исклочить модуль из архива. Заголовок пункта задания имеет вид

$$
+\operatorname{DELETE}\left\langle\begin{array}{c}
\text { тип } \\
\text { модуля }
\end{array}\right\rangle:\left\langle\begin{array}{c}
\text { архивное имя } \\
\text { модуля }
\end{array}\right\rangle
$$

Телг данного пункта пусто.

Пример. В результате выполнения пункта задания

+DELETE PRUNIT : OLMU 04

модуль программная единица с именем OLMU 04 будет из архива исклпочен.

\section{Вставить текст. (Предложение INSERT)}

При работе с панетом часто оказывается полезным выделить неготорые части, общие для нескольких модулей, и рассматривать их как самостоятельные объекты. Такое выделение можно реализовать посредством модуля типа MAORO, слуякашего для выполнепия магроподстановок.

Пример. Блго общей памнти COMBAS в модуле OLMU 04 нужен не тольто для подпрограмм RVAR, но и для целого ряда других подпрограмм. Выделим его в модуль типа MACRO: 
1 ALTIME, CPTIME, NLEDGE, NLEND, NLRES, NONLIN,

2 NOUT, NPRINT, NREAD, NREC, NRESCM, NSTEP LOGICAL

1 NIJEND, NLRES

Если некоторая часть текста модуля оформлена в виде другого модуля, то перед выходом на выполнение модуля, например, на трапсляцию программной единицы, система должна проиэвести вставку в текст модуля. Указание на необходимость вставок осуществляется предложением INSERT, имегоим формат, аналогичный формату заголовка пунгта задания:

$$
+\operatorname{INSERT}\left\langle\begin{array}{c}
\text { тип } \\
\text { модуля }
\end{array}\right\rangle:\left\langle\begin{array}{c}
\text { Функциональное имя } \\
\text { модуля }
\end{array}\right\rangle
$$

Предложение INSERT должно быть записано в теле модуля в том месте, в котором нуянно произвести вставку.

Пример. Модуль OLMU 04 после оформления модуля OLMC 11 можно записать в архив следугщим образом:

+STORE PRUNIT : OLMU 04

SUBROUTINE RVAR(KNAME, PVALUE)

$\mathrm{C}$

С 0.4 ПЕЧАТЬ ИДЕНТИФИКАТОРА И ЗНАЧЕНИЯ

C ПЕРЕМЕННОЙ

$\mathrm{C}$

+INSERT MACRO : OLMC 11

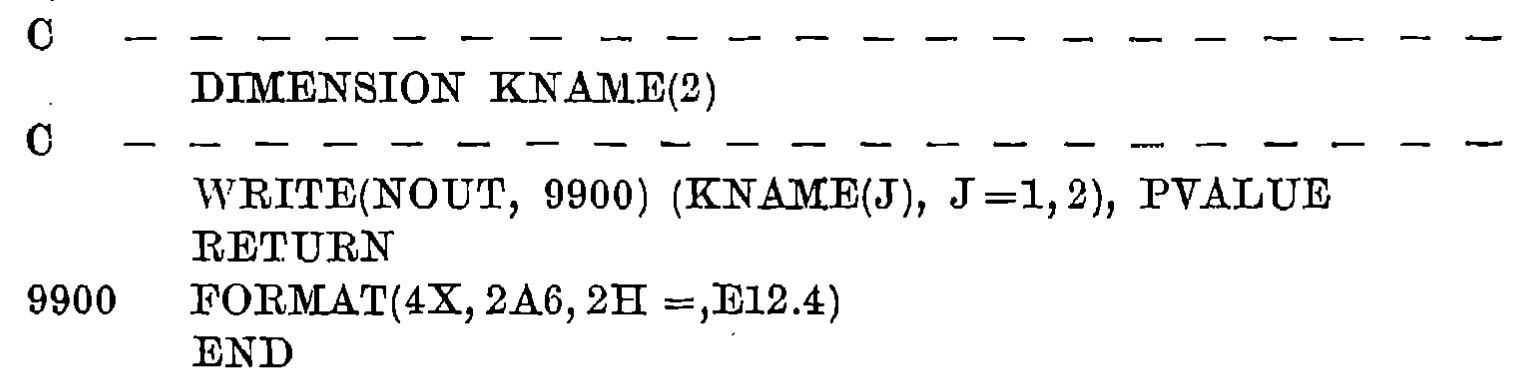

Преимущество аппарата вставон заклочается в том, что подстановка участка текста производится системой только при выполнении модуля. Поэтому, например, замена вставки приводит фактически и иэменению целого ряда модулей, пмеюших ссылку на эту вставку.

Использование вставок позволяет выделить и обрабатывать кан самосгоятельные модули такие части программ, оформление которых в виде отдельных программных единиц, допускающих автономную трансляцию, нежелательно по каким-либо причинам. (Например, из-за синтаксических затруднений или же из соображений экономии расхода машинного времени на вызовы программных единиц). Кроме 
того, вставіки, в совокупности с модуллми типа вариант, представляюо собой доволыю гибюе средство формировашия тскстов из набора написанных ранее макрочастей.

\section{Работа с вариантом. (Предложенше МАТСН)}

Одной из важнейних особенностей пакета является возможность состанления различных вариантов сложных программ из существуюшцего набора модулей. Основным средством описания нонгретных вариантов программ являются предложения МАТСЕ.

Предложения МАTСН устанавливапот соответсгвие менду имепами, содернкащимися в предлонениях INSERT (т.е. (унпционалыпыми имепами) с одно ің стороны и именами модулей в архиве - с другой.

Предложепие МАТСН имеет формат

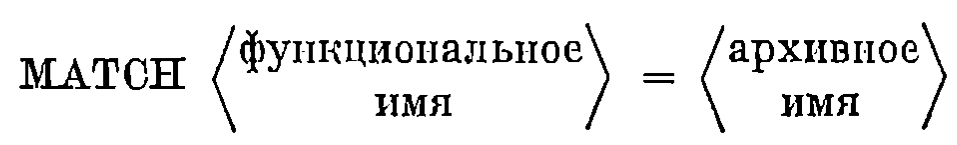

Приате. Пусть в программе имеется обрашение г подпрограмме RVAR, описанной в приведенном ранее примере. Вообще говоря, в архиве пакета может храниться несколько программных единиц с одним и тем же фортранным именем RVAR, но, естественно, с различными архивными именами. Если нам нуяно использовать подпрограмму RVAR, оформленпуг в виде модуля типа PRUNIT под именем OLMU 04, мы должны записать следующее предложение

$$
\text { MATCH RVAR = OLMU } 04 .
$$

Последовательность предложений МАТСЕ мояюно оформить в виде отдельного модуля типа вариант.

Прнмер. Пусть основная программа эаписана в архив следующим бразом :

+STORE PRUNIT: OLM 000

PROGRAM MAIN

C

C 0.0 OCHOBIAЯ ПРОГРАMMA

$\mathrm{C}$

+ INSERT MIACRO : COMBAS

$\mathrm{C}-\ldots-\ldots-\ldots-\ldots$

$\mathrm{C}$

C BPEMЯ, ЗАГАЗАННОЕ ДЛЯ РАБОТЫ

CALL JOBTIM(ALTIME) 


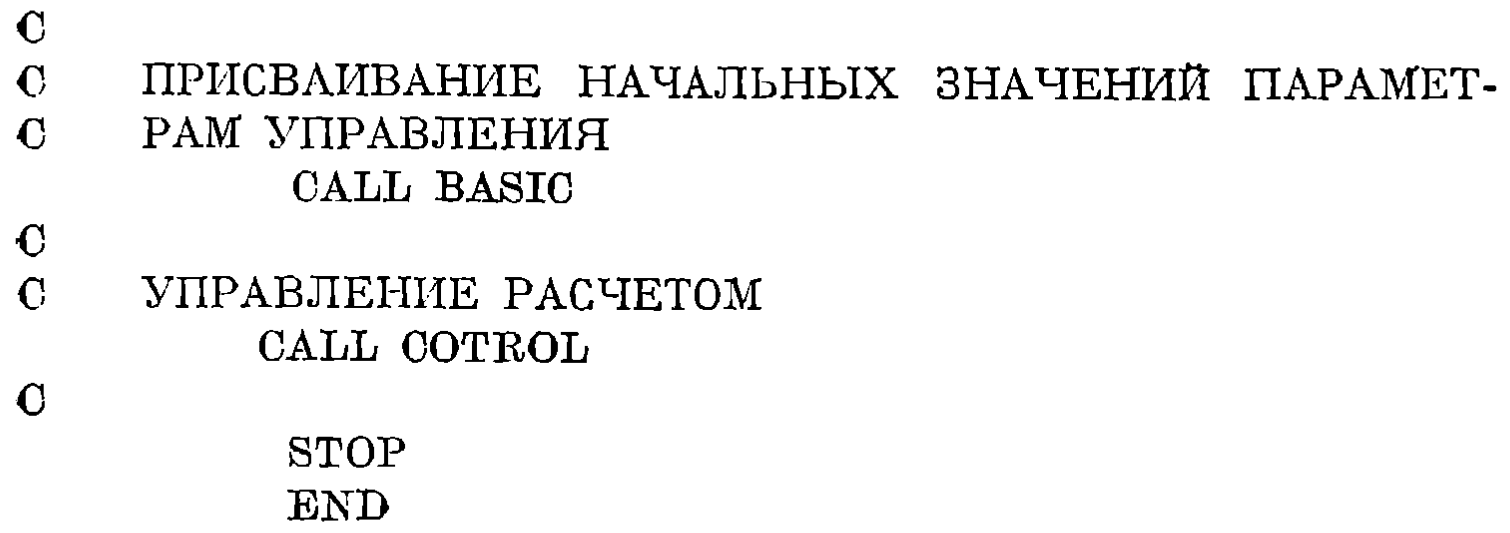

Она содеринт обращение $k$ подпрограммам JOBTIM, BASIO, COTROL, которые хранятся в архиве под именами OLMU 25, OLM001 и OLM 002. Мы можем составить следующий модуль, описывающий вариант сборки программы (строни, начинагощиеся с $+\mathrm{C},-$ это строки томментария):

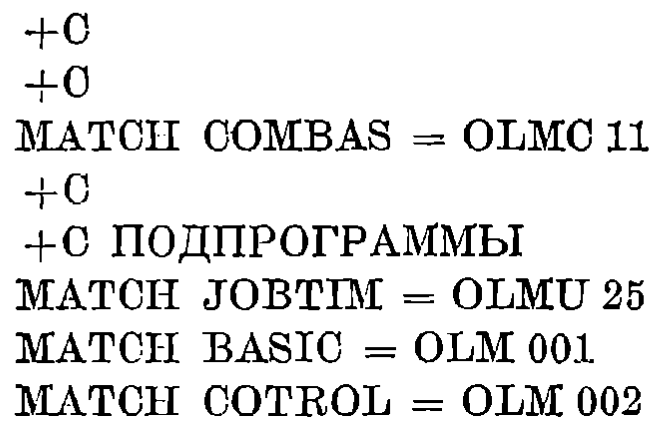

Если имя, содержащееся в операторе вызова программной единицы языка программирования или в предложении INSERT, не встречается ни в одном предложении МАТСН, оно рассматривается хак имя модуля в архиве. Такнм образом, уточнение функциональных имен заменой их на архивные пмена может производиться либо явно, посредством предложения МАТСН, либо неявно, по умолчанию, когда функциональное имя совпадает с архивным.

Еслі некоторое фунгциональное имя встретается в левых частях нескольких предложений МАТСН, то ему в соответствие ставится архивное имя, указанное последним. Поэтому модуль типа вариант, выполияясь после модуля, задатощего версио, детализацией которой он является, может вносить изменения в эту версиг, предписывая своими предложениями МАТCЕ некоторые замены функциональных имен на архивные пмена, отличающиеся от тех архивных имен, которые ставятся в соответствие тем же функциональным именам предложениями МАТСН, содержащимися в модуле версии. 


\section{6. Общая схема обработки задания}

Выполпением задапия для системы САФРА управляет монитор, представзялщий собой совокупность программных средств, обеспечиваюџих операциониыс возможности панета. Эти средства базируются на штатном программном обеспсчении вычислительной машины, т.е. с системной точюи монитор панета имеет статус обычной задачи. Монитор интерпретирует задание пункт за пунктом, выполняя работы, предписываемые гажғдым очередным пунктом задания до тех пор, пюка не будет исчерпано все задание, либо пока не встретится накой-либо „кримипальной" пункт, выполнение готорого огаяется невозможным из-за его некорректного обормления, либо из-за песоответствия реальному состоянию архива.

Основными компонентами системного наполнения пакета являоотся : интерпретатор языка заданий, препроцессор текстов, внугренняя информационная служба, генератор заданий для мониторной системы „Дубна". Отметим, что в языке заданий нет понятия модуля загрузки, поскольу пакет избавляет пользователя от необходимости оперировать этим понятием п берет на себя всп службу по организации хранения, учета и использования транслированных программных едпниц. При выполнении каждого нового задания те конфигурации модулей, ноторые транслировались ранее, не транслируются заново, а вместо них из системы хранения автоматичесіи подставляготся оттранслгрованные программные единицы.

Выполняя заданне пользователя, монитор взаимодействует с архивом системы, организуя внесение туда изменений, а также посылает запросы на выборку из архива нужной информации. Он гонтролирует и другие внутрисистемные связи, посылая задания па распечатки тегстов и взаимодействуя с информационной и планирующей слуякбами системы. Собственная функциональная деятельность монитора состоит в выполнении предписанных процедур по сборне с угазанной в заданип заменой фунгциональных имеп модулей на архивные имсна. Fроме того, монитор органнзует внсшние связи системы с ғомпонентами штатного математического обеспечения ЭВМ (операнионой спстемой, тралсляторами и т.д.).

\section{7. Ииформационная система}

С точки зрения пользователья пакет принладных программ есть вместилище зианий об алгоритмах решения задат из данпой предметной области. Однатю, зачастуго воспользоваться этими зианиями бывает очень сложно, посгольку информация о предметпой области іл дисциплине работы с программным фондом развитого пакета очень обширна, 
а по своей стругтуре чрезвычайно разнородна. Для работы с пакетом пользователю приходится изучать объемную программнуго документацию, вследствие чего эффект использования панета значительно уменьшается, так как эта работа бывает порой сравнума с работой по созданию вычислительного алгоритма. Для того, чтобы достигнуть высокого уровня организации работ при проведении вычислительного эксперимента средствами пакета прикладных программ, необходимо создать средства пиформационного обслуживания пакета, позволяющие накапливать „знания о знаниях" п производить логическуг обработну информации, связанной с проведением вычгслительного энсперимента.

Информационная система пакета САФРА решает четыре следующие основные задачи:

1. Задача ознакомления со структурой пакета. Цель этой задачи заключается в том, птобы пользователь, впервые обратившийся н информационной системе, имелг возможность выяснить, описания наних объектов заложены в информационнуго базу и каковы наборы характеристик каждого объента, или, другими словами, мог выяснить что л о чем можно спрашивать у системы.

2. Задача изучения характеристик объектов. Эта эадача наиболее важна с практической точки эрения. Действительно, основными объектами, которыми оперирует пакет САФРА, являгося архивные модули. Уже сейчас архив пакета содержит около 1000 модулей, основную часть готорых составлягот модули типа программная единица. Хотя модули типа схема счета, версия, вариант, вставка составляго меньшую часть, но и они песут значительную функциональную нагрузну. Большую долю всех вопросов, возникающих у пользователя в процессе эксплуатации пакета, составляют вопросы типа: наковы эксплуатационные харагтеристики данной программной единицы, гағово назначение данной схемы счета и из паких модулей она состоит, чем харантеризуется данный вариант программы, гакие изменения локализованы в текстовой вставке и т.д. Цель решения задачи изучения характеристик объектов в первуго очередь состопт в получении ответов на эти и аналогичные вопросы относительно характеристик архивных модулей. Попутно при этом решается задача слежения за текущим состояннем архива, что является важным для организации архивной службы пагета.

3. Задача ознакомления со структурой программы и структурой ее данных. При желании пользователя подробно ознатомиться с программной реализацией алгоритма, он должен иметь возможность получить ответы на вопросы типа: 
- в гагом модуле запрограммирован тот или иной алгоритм;

- откуда вызывается данная программная единица;

- гаковы входные п каловы выходные пгараметры данного модуля ;

- где определяется и где используется в программе данная переменная, и так далее.

Подобная информация нуяна в частности при попытке нспользовать имеощийся фонд пакета для создапия повой версии программы.

4. Задача конструирования программы из модулей функционального наполнения. Системә, решапоая эту пнформациопнуло задачу, представляет собой собеседника, который осведомлен о том, папие схемы счета, версии и варианты программ имеготся в архиве пағета, и какие математические модели они реализугт. Она также, имея детальное представление о назначении и устройстве программных единиц, отвечая на вопросы пользователя, поможет ему выбрать и віллочить в вычислительнуго цепочку необходимый алгоритм. При этом формпрование задания для панета на выборну и включение модуля в цепочку может производиться самой инфформационной системой, так qто полученная программа будет заведомо корректной в смысле программирования.

\section{Oпыт эксплуатации пакета}

Приведем некоторые статистические данные. Программный фонд, хранящийся в архиве САФРА, содержит в настоящее время свыше 1000 модулей суммарным обтемом свыше 70000 предложений различ ных алгоритмических языков. За два года эгсплуатации пакета проведены расчеты более 8000 вариантов программ.

Практика работы с пакетом показала, что его применение имеет определенные преимущества по сравнению с использовавшейся ранее организацией работ по нонструированио больших программ. В частности, раньше существовал некоторый порог в размерах программы, при переходе через который разработчик ее должен был „связать с ней свого судьбу", т.е., с одной стороны, программа не могла эфффективно эксплуатироваться без разработчика, а с другой стороны, основная часть трудовоћ деятельности разработчика посвяшаласт сопровокдению программы, внесению в нее изменений и организации расчетов гонкретных вариантов. Использование пакета САФРА позволило в значительной мере преодолеть упомянутые трудности. 


\title{
Литература
}

[1] В. Я. Iіарпов, Д. А. Ііорягин, А. А. Самарский, Принұипы раврабопћи пакешоо принладных програм.и д.и задач математической бизики, Ж. вытисл. мат. II мат. фин. 18 (1978), 458.

[2] K. V. Roberts, An introduction to the OLFMLOS system, Comp. Phys. Communications 7 (1974), 237.

[3] J. P. Christinnsen, K.V. Roberts, OLIMPUS, a standard control and utility package for intial-value Fortran Programs, ibid. 7 (1974), 245.

[4] II. М. Горбунов-Посадов, В. Я. Карпов, Д. А. Коряги, В. В. Kра.

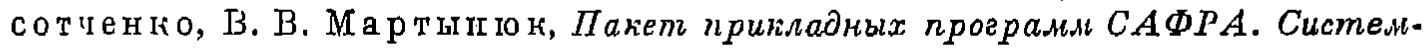
иое наполиение, Препринт ИПМ АН СССР 85 (1977).

[5] M. М. Горбунов-Посацов, Л. А. Хиздер, Система ОХРA. Основные понлmun. Baзовые операуии, Преприпт IIIM АН CCCP 67 (1979).

[6] -, -, Работа с регионами и дайлами в системе ОХРА, Препринт ИПМ АН CCCP 81 (1979).

[7] В. Я. Карпов, В. В. Красотченко, Задачи ипрорлачионново обеспеченил оыиислительного әксперимента, Препринт ИПМ АН СССР 127 (1979).

[8] С. А. Га йфулин, В. Я. К арпов, Т. В. Ми щенк о, САФРА. Фуккииональное папо.инене, система OLYMPUS, Препринт ИПМ AH GCCP 27 (1980).

[9] M. М. Горбунов-Посадов, Д. А. Корягии, В. В. І расотченко, Реализаиил системного наполиения пакета прикладних програмя САФРA (Версил 2.0), Препринт ИПМ АН CGGP 186 (1979).

\author{
Presented to the Semester \\ Computational Mathematics \\ February 20-May 30, 1980
}

